\title{
THE EFFECT OF BISPHOSPHONATE TREATMENT ON THE BIOCHEMICAL AND CELLULAR EVENTS DURING BONE REMODELLING IN RESPONSE TO MICROINJURY STIMULATION
}

\author{
L.E. Mulcahy ${ }^{1,2, \dagger}$, C.M. Curtin ${ }^{1,2,3, \dagger}$, R.J. McCoy ${ }^{1,2}$, F.J. O’Brien ${ }^{1,2,3}$, D. Taylor ${ }^{1,3}$, T. Clive Lee ${ }^{1,2}$ and G.P. Duffy ${ }^{1,2,3, *}$
}

\begin{abstract}
${ }^{1}$ Trinity Centre for Bioengineering, Department of Mechanical Engineering, Dublin, Ireland ${ }^{2}$ Department of Anatomy, Royal College of Surgeons in Ireland, St. Stephen's Green, Dublin, Ireland ${ }^{3}$ Advanced Materials and Bioengineering Research (AMBER) Centre, RCSI \& TCD, Dublin, Ireland
\end{abstract}

${ }^{\dagger}$ Both authors contributed equally to this work

\begin{abstract}
Osteoporosis is one of the most prevalent bone diseases worldwide and is characterised by high levels of bone turnover, a marked loss in bone mass and accumulation of microdamage, which leads to an increased fracture incidence that places a huge burden on global health care systems. Bisphosphonates have been used to treat osteoporosis and have shown great success in conserving bone mass and reducing fracture incidence. In spite of the existing knowledge of the in vivo responses of bone to bisphosphonates, the cellular responses to these drugs have yet to be fully elucidated. In vitro model systems that allow the decoupling of complex highly integrated events, such as bone remodelling, provide a tool whereby these biological processes may be studied in a more simplified context. This study firstly utilised an in vitro model system of bone remodelling and comprising all three major cell types of the bone (osteocytes, osteoclasts and osteoblasts), which was representative of the bone's capacity to sense microdamage and subsequently initiate a basic multicellular unit response. Secondly, this system was used to study the effect of two commonly utilised aminobisphosphonate treatments for osteoporosis, alendronate and zoledronate. We demonstrated that microinjury to osteocyte networks being treated with bisphosphonates modulates receptor activator of nuclear factor kappa-B ligand and osteoprotegerin activity, and subsequently osteoclastogenesis. Furthermore, bisphosphonates increased the osteogenic potential following microinjury. Thus, we have shown for the first time that bisphosphonates act at all three stages of bone remodelling, from microinjury to osteoclastogenesis and ultimately osteogenesis.
\end{abstract}

Key Words: Bone remodelling, basic multicellular unit, microinjury, osteoclastogenesis, osteogenesis, osteoporosis, bisphosphonate treatment.

*Address for correspondence:

Garry P. Duffy, BSc, PhD

Department of Anatomy

Royal College of Surgeons in Ireland

123 St. Stephens Green

Dublin 2, Ireland

Telephone Number: +35314022105

FAX Number: +355 14022355

E-mail: garryduffy@rcsi.ie

\section{Introduction}

Osteoporosis is one of the most prevalent bone diseases worldwide and is characterised by high levels of bone turnover, a marked loss in bone mass and the accumulation of microdamage. Together, these factors culminate in a reduced bone density and altered osseous microstructure, leading to a decrease in the mechanical competence of bone and an increasing susceptibility to fracture (Parfitt, 2002). In 2000, it was estimated there were approximately 9 million osteoporosis related fractures globally each year (Johnell and Kanis, 2006). In 2005, based on data from the previous 25 years (Chrischilles et al., 1994; Hoerger et al., 1999, Phillips et al., 1988, Ray et al., 1997), it was estimated that there would be over 2 million osteoporosis related fractures annually in the United States at a cost of $>\$ 17$ billion, which would grow to greater than 3 million fractures per annum at a cost of $>\$ 25$ billion by 2025 (Burge et al., 2007; Roche et al., 2005). Osteoporosis is a common condition observed in both elderly men and women; however, women have a four times greater risk of developing osteoporosis, with $30 \%$ of postmenopausal women worldwide being affected. The discrepancy between sexes is believed to be associated with changes in oestrogen content, whereby oestrogen deficiency has been associated with increased bone resorption due to an increase in activated osteoclasts (Eriksen et al., 1999) but also an increase in bone formation that is unable to compensate for the enhanced resorption (Brennan et al., 2011; Jilka et al., 1998).

Whilst bone remodelling (or bone turnover) may be influenced by systemic hormones, such as oestrogen and parathyroid hormone (Donahue et al., 2006), other factors such as mechanical loading are also known to contribute to osteoporosis as it is a major regulator of bone mass and geometry with the sensing and transduction of strains being essential for the maintenance of healthy bones (Bergmann et al., 2010). Individual bones within the skeleton experience different mechanical loads, not only as a function of their location, but also in response to the level of physical activity (Burr et al., 1996), age (Nordin et al., 1990) or environment (Zerwekh et al., 1998) of an individual. Conditioning of bone to withstand these forces occurs through dynamic remodelling that aims to minimise stress or strain concentrations (Lanyon, 1987) and provide an optimal mechanical structure. Stimulation or activation of bone remodelling has been attributed to factors such as fluid flow (Kufahl and Saha, 1990; Weinbaum et al., 1994) and microdamage (Burr et al., 1985; Lee et al., 
2002; Martin and Burr, 1982) within the bone, which is hypothesised to be sensed by osteocytes either through mechanotransduction mechanisms (Turner et al., 1994) or by the shearing of cellular processes (Hazenberg et al., 2009; Taylor et al., 2003). This signalling mechanism, between mechanically stimulated osteocytes and osteoblast progenitors within the bone marrow, is believed to be paracrine in nature as osteocytes are spatially isolated from the marrow cavity (Hoey et al., 2011). Moreover, regions of osteocyte apoptosis have been shown to be accompanied by increased local bone turnover and targeted bone resorption and are believed to be involved in osteoclast precursor recruitment and differentiation in vitro (Al-Dujaili et al., 2011). Subsequently, the receptor activator of nuclear factor kappa B ligand-receptor activator of nuclear factor kappa B- osteoprotegerin (RANKL-RANK-OPG) signalling pathway is activated (Mulcahy et al., 2011), and in turn mediates the initiation of bone remodelling through the synchronous action of the basic multicellular unit (BMU) that consists of the main cell types involved in bone remodelling: osteoblasts, osteocytes and osteoclasts. Accumulation of microdamage within the bone due to excessive overloading (Burr et al., 1998), the impairment of osteocyte functionality, or perturbations in the equilibrium of the resorption and formation arms of the BMU (Mashiba et al., 2000), as in osteoporosis, increases an individual's susceptibility to fragility fractures due to diminished structural integrity or loss of bone mass and thus the mechanical competence of bone.

Bisphosphonates, synthetic analogues of inorganic pyrophosphate, containing a phosphate-carbon-phosphate backbone, have been used as drugs to treat osteoporosis for the last 30 years (Morris and Einhorn, 2005). They act as potent inhibitors of bone resorption, in an effort to conserve bone mass and thereby effectively reduce the risk of osteoporotic fracture, by the prevention of pre-osteoclast recruitment, fusion and differentiation (Russell et al., 2008). They function by binding tightly to bone mineral, where they are internalised by osteoclasts by the process of endocytosis during bone resorption. Here, they inactivate osteoclast function and induce osteoclast apoptosis. Bisphosphonate-treated bone is characterised by fewer osteoclast cell numbers, most of which are located away from the bone surface, and many of which are undergoing apoptosis (Pivonka et al., 2012). Bisphosphonates are not metabolised and therefore are either excreted renally or bind to hydroxyapatite within the bones where they can remain for years (Reginster et al., 2013). This skeletal binding affinity has been shown to increase in rank order through risedronate, ibandronate, alendronate and zoledronate, with higher affinity bisphosphonates becoming rebound more quickly thereby increasing skeletal retention (Russell et al., 2008). Many studies have reported that bisphosphonate treatment increases the degree of mineralisation in bone, by facilitating the binding of hydroxyapatite crystals onto bone surfaces, principally at the sites of bone remodelling (Boivin et al., 2000; Rogers, 2003; Spadaro et al., 2006).

In vitro model systems that allow the decoupling of complex highly integrated events, such as bone remodelling, provide researchers with a tool by which biological processes may be studied in a more simplified context.
This will allow researchers to expand the knowledge space pertaining to the interactions between individual components of the biological system and secondly, create a test bed for studying how current treatments exert their observed clinical effects and how potential therapeutic treatments may modulate inherent biological functions. This study aimed to utilise a previously established in vitro model system of bone remodelling, that comprises all three major cell types of the bone (osteocytes, osteoclasts and osteoblasts), in which osteocyte networks can be subjected to controlled amounts of microinjury, allowing the investigation of bone's capacity to sense damage and initiate a BMU response (Mulcahy et al., 2011). We have applied this system to assess the effect of alendronate and zoledronate, two aminobisphosphonate treatments for osteoporosis that exhibit the highest affinity for bone mineral, on the osteocytic response to microdamage and the subsequent impact on BMU activation.

\section{Materials and Methods}

\section{Cell culture}

MLO-Y4 cells, an osteocyte-like cell line originally derived from murine long bones (Kato et al., 1997) were generously provided by Dr. Linda Bonewald (School of Dentistry, University of Missouri, Kansas City, MO, USA), and were maintained in $\alpha$ Modified Eagle's Medium ( $\alpha$ MEM; Biosera, Uckfield, UK) supplemented with $5 \%$ foetal bovine serum (FBS; Biosera, East Sussex, UK), $5 \%$ iron supplemented calf serum and $1 \%$ penicillin/ streptomycin (P/S; Sigma Aldrich, Dublin, Ireland). Cells were cultured in collagen coated flasks $(0.15 \mathrm{mg} / \mathrm{mL}$ rat tail collagen type 1 ; BD Biosciences, Oxford, UK) at $37^{\circ} \mathrm{C}$ and $5 \% \mathrm{CO}_{2}$.

Pre-osteoclasts (RAW 264.7 cells, ATCC, LGC Standards, Teddington, UK) were cultured in high glucose Dulbecco's Modified Eagles Medium (DMEM; Sigma Aldrich) supplemented with $10 \%$ heat inactivated FBS (Biosera) and $1 \% \mathrm{P} / \mathrm{S}$.

Pre-osteoblasts (MC3T3-E1 cells, ATCC, LGC Standards, Middlesex, UK) were maintained in aMEM supplemented with $10 \%$ FBS, $1 \%$ L-glutamine (Sigma Aldrich), and $2 \% \mathrm{P} / \mathrm{S}$. 20 .

All cell lines were used in experiments below passage

\section{Generation of osteocyte microinjury and conditioned medium samples}

Conditioned media was obtained following application of microinjury to gel-embedded osteocyte (MLO-Y4) cells, as previously described (Mulcahy et al., 2011). Briefly, constructs were prepared by the addition of 1 volume of Matrigel Basement Membrane Matrix (BD Biosciences) to 1 volume of a collagen solution comprised of $58 \%$ Collagen, $27 \% 5 \times$ DMEM, $2.5 \%$ FBS, $2.5 \%$ calf serum, $5 \%$ sodium hydroxide (Sigma Aldrich) and $5 \%$ cell suspension. An incubation of $5 \mathrm{~d}$ was sufficient to facilitate cell process network formation. Microinjuries were applied through insertion of needles of diameter 160, 300 and $400 \mu \mathrm{m}$ into the centre of the hydrogel, creating a planar 
defect by drawing the needle through the hydrogel at a right angle to the point of insertion to create a defect $7 \mathrm{~mm}$ in length and $5 \mathrm{~mm}$ in depth. After $72 \mathrm{~h}$ of culture, conditioned medium samples were collected. The term microinjury has been applied to the planar defects created in this study, which are intended to be reflective of microdamage, but due to acknowledged differences between the in vitro cultures compared to the in vivo scenario, such as osteocyte density and process number per osteocyte, we have refrained from using the word "microdamage" to represent the defects created, as they are not truly of a length scale equating to microdamage. The individual cell types involved in bone remodelling were spatially decoupled by cultivating them separately. Cellular crosstalk was evaluated through transfer of conditioned medium from the 3D gel-embedded osteocyte (MLO-Y4 cells) network cultures, $72 \mathrm{~h}$ post microinjury, to the osteoclast (RAW 264.7 cells) monolayer cultures, which was then in turn serially transferred to pre-osteoblast (MC3T3 cells) monolayer cultures to yield doubly conditioned media. Media were also assessed in all experiments from gel-embedded osteocytes not subjected to injury, termed uninjured cells (i.e. the negative control).

For all experiments assessing the effects of bisphosphonate treatment on pre-osteoclasts and preosteoblasts, $10^{-5} \mathrm{M}$ concentrations of alendronate or zoledronate were added to the culture medium only at the time of osteocyte seeding on the gels. Conditioned and doubly conditioned media were then taken as described previously for treatment of the pre-osteoclast and preosteoblast cells.

\section{Cell migration assays}

In order to assess whether osteocyte injury or osteocyte injury/osteoclastogenesis, with and without $10^{-5} \mathrm{M}$ bisphosphonate treatment, stimulates the migration of pre-osteoclast and pre-osteoblast cells respectively towards a site of damage, a cell migration assay was performed. This assay is based on the concept that following a period of serum starvation, cells will migrate through a porous membrane towards a stimulus. Under serum free conditions, $1 \times 10^{4}$ pre-osteoclasts/pre-osteoblasts were cultured on the upper side of $8 \mu \mathrm{m}$ pore hanging cell culture inserts (Millipore, Cork, Ireland). Following a $2 \mathrm{~h}$ incubation, serum free medium was replaced with conditioned/doubly conditioned medium from injury experiments $(n=3$ per injury size). Media were also assessed from gel-embedded osteocytes not subjected to injury, termed uninjured cells. Controls were serum free medium (OptiMEM; Invitrogen, Dublin, Ireland) and cell culture medium containing $10 \%$ FBS. Cells were incubated for $18 \mathrm{~h}$, following which cellseeded membranes were fixed in $4 \%$ formalin for $10 \mathrm{~min}$, and stained with haematoxylin for $10 \mathrm{~min}$. The membrane was then removed and mounted on a glass slide, bottom side down. Cell migration was quantified by counting the cells on the bottom side of the membrane in 5 random fields ( $n=3$ per treatment group).

\section{Osteoclastogenesis}

Pre-osteoclasts were seeded at a density of $4 \times 10^{4}$ cells/ well in 12-well adherent plates (Cruinn Diagnostics,
Dublin, Ireland) or on poly-D-lysine (1 M)-coated chamber-slides. Pre-osteoclast cell culture medium was replaced with osteocyte conditioned medium, with or without $10^{-5} \mathrm{M}$ bisphosphonate treatment, $24 \mathrm{~h}$ after seeding. Conditioned medium was changed every 3 days. After $21 \mathrm{~d}$, tartrate-resistant acid phosphatase (TRAP) activity was quantified ( $n=3$ per treatment group) by removing the culture medium from pre-osteoclast cultures and incubating it for $1 \mathrm{~h}$ in $1 \mathrm{mM}$ citrate solution at $37^{\circ} \mathrm{C}$. This culture medium solution $(20 \mu \mathrm{L})$ was then added to $50 \mu \mathrm{L}$ of a solution containing $100 \mathrm{mmol}$ p-nitrophenyl phosphate (pNPP) (Sigma Aldrich), 200 mmol sodium citrate (Sigma Aldrich, Dublin, Ireland) and $80 \mathrm{mmol} / \mathrm{L}$ sodium tartrate (Sigma Aldrich) in a 96-well plate, and incubated for a further $30 \mathrm{~min}$ at $37^{\circ} \mathrm{C}$. NaOH $(20 \mu \mathrm{L}$ of $1 \mathrm{M}$ solution) was then added to stop the reaction and absorbance was read at $405 \mathrm{~nm}$ by spectroscopy. TRAP staining was performed using a Leukocyte Acid Phosphatase Kit (Sigma Aldrich) in accordance with the manufacturer's instructions. Furthermore, the number of TRAP positive multinucleated cells were quantified, from 3 random fields of view, at $20 \times$ magnification, and their frequency expressed at a percentage of cells within the field of view ( $n=3$ per treatment group).

\section{Osteoblast differentiation}

Pre-osteoblasts were seeded at a density of $4 \times 10^{4}$ cells in 6 well plates. Cells were allowed to attach for $24 \mathrm{~h}$ prior to replacing growth medium with a $1: 1$ ratio of doubly conditioned medium from microinjury/ osteoclastogenesis experiments, with or without $10^{-5} \mathrm{M}$ bisphosphonate treatment, combined with either growth or osteogenic medium. Osteogenic medium was prepared by the addition of ascorbic acid $(50 \mu \mathrm{g} / \mathrm{mL}$; Sigma Aldrich), $\beta$-glycerophosphate (10 mM; Sigma Aldrich) and dexamethasone (100 nM; Sigma Aldrich) was added to standard $\alpha \mathrm{MEM}$ supplemented with $10 \% \mathrm{FBS}, 1 \%$ L-glutamine and $2 \%$ penicillin/streptomycin. Cells were cultured for $21 \mathrm{~d}$ at $5 \% \mathrm{CO}_{2}$ and $37^{\circ} \mathrm{C}$.

To assess osteogenic differentiation, treatment medium was removed from pre-osteoblasts cultured for $21 \mathrm{~d}$, cells were rinsed in phosphate buffered saline (PBS; Sigma Aldrich) and $500 \mu \mathrm{L}$ of $1 \mathrm{M} \mathrm{HCl}$ added to each well. Cells were manually detached using a cell scraper and the resulting cell suspension placed into a $1.5 \mathrm{~mL}$ cryovial. Wells were then washed a second time with an additional $500 \mu \mathrm{L}$ of $1 \mathrm{M} \mathrm{HCl}$ and this was added to the initial cryovial. Samples were left overnight at $4{ }^{\circ} \mathrm{C}$. Calcium deposition was quantified using the Calcium Liquicolor Kit (Stanbio Laboratories, Boerne, TX, USA) according to the manufacturer's protocol and calcium concentration was deduced using a standard curve $(n=3$ per treatment group).

\section{Effect of bisphosphonate treatment on RANKL and OPG expression levels after osteocyte microinjury}

Studies were performed to determine an optimum dose of bisphosphonate treatment (data not shown). As a result, a $10^{-5} \mathrm{M}$ concentration of each bisphosphonate, consistent with concentrations suggested in the literature (Naidu et 


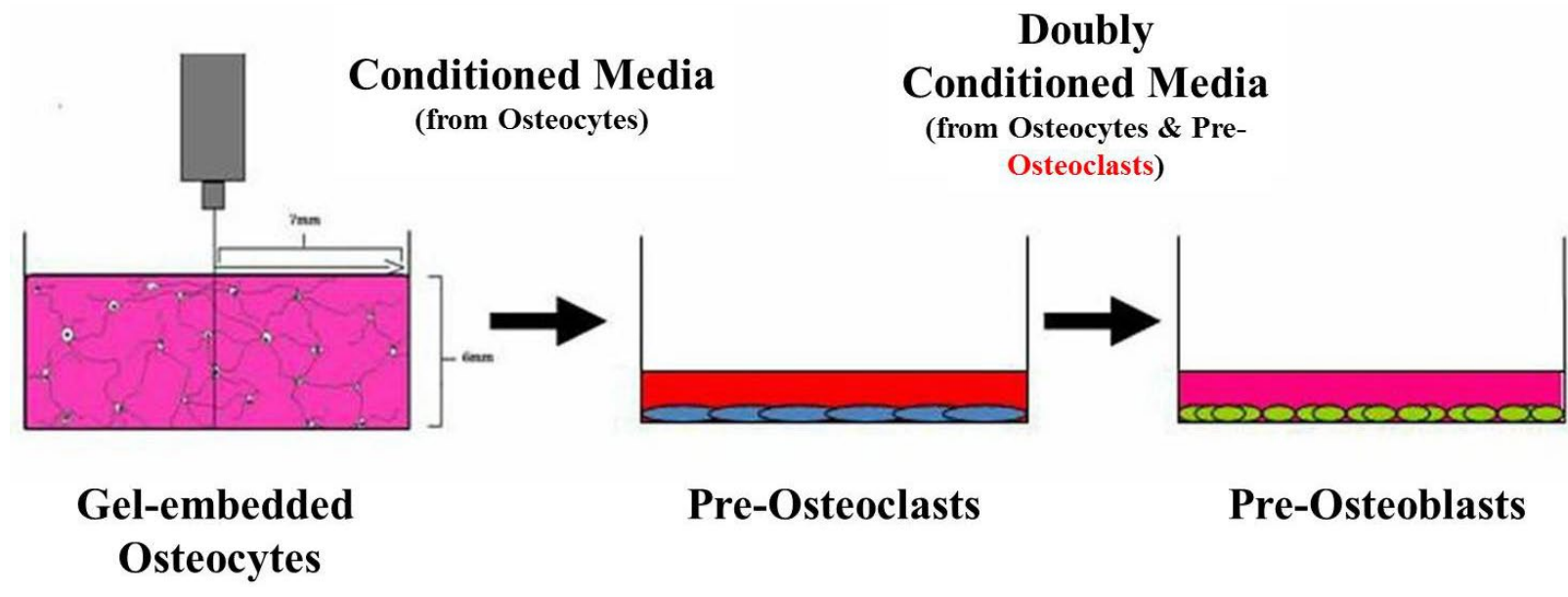

Fig. 1. Diagrammatic representation of the in vitro bone remodelling system for studying the relationship between osteocyte signalling in response to microinjury and BMU activation and function. The individual cell types involved in bone remodelling are spatially decoupled by cultivating them separately. Cellular crosstalk is then evaluated through transfer of conditioned medium from the 3D gel embedded osteocyte (MLO-Y4 cells) network cultures, post microinjury, to the pre-osteoclast (RAW 264.7 cells) monolayer cultures, which are then in turn serially transferred to pre-osteoblast (MC3T3 cells) monolayer cultures.

al., 2008), was chosen to be the optimum dose to maintain osteocyte viability whilst still stimulating a temporal pharmacological response.

Gel-embedded osteocytes were incubated using OptiMEM serum-free medium, supplemented with $10^{-5} \mathrm{M}$ concentration of both alendronate and zoledronate. Samples were taken at $24 \mathrm{~h}$ intervals over a $72 \mathrm{~h}$ time period. RANKL and OPG were quantified by means of single site specific ELISA (Mouse RANKL and OPG ELISA, R\&D Systems) under manufacturers' instructions. Cultures $(n=3)$ were assayed at each injury level, in addition to non-injured controls.

\section{Statistical analysis}

All experiments contained $n=3$ technical repeats and were performed in triplicate. All data were analysed for significance $(p \leq 0.05)$ using one way ANOVA followed by a post-hoc Tukey test, to compare means between injured and uninjured groups and between drug treated and non-drug treated groups under the same experimental parameters.

\section{Results}

\section{Development of an osteocyte-BMU in vitro model}

The initial set of studies aimed to identify the required microinjury size needed in the gel-embedded osteocyte networks to yield conditioned medium capable of recruiting and differentiating pre-osteoclasts, which in turn, would be capable of recruiting pre-osteoblasts and yielding increased mineralisation when using doubly conditioned media (media exposed to osteocytes and pre-osteoclasts), thereby mimicking the in vivo scenario. Fig. 1 represents a diagrammatic representation depicting the in vitro bone remodelling system designed to study the relationship between osteocyte signalling in response to microinjury and BMU activation and function.

The effect of microinjury size to osteocyte networks on pre-osteoclast migration and differentiation

Conditioned media were taken from gel-embedded osteocyte networks, subjected to microinjury widths of 160,300 or $400 \mu \mathrm{m}$ in addition to uninjured cells after $72 \mathrm{~h}$, and added to pre-osteoclasts to assess migration and differentiation potential. A significant increase in preosteoclast cell migration was observed, in response to the conditioned medium from microinjured osteocyte networks that experienced injury widths of 300 and $400 \mu \mathrm{m}$, in comparison to the smaller injury width or the undamaged control (Fig. 2a). Osteoclastogenesis, as measured by TRAP activity, was significantly increased for all injury sizes in comparison to the undamaged control (Fig. 2b). TRAP staining of pre-osteoclasts suggested the presence of larger multinucleated cells was greatest in $300 \mu \mathrm{m}$ and $400 \mu \mathrm{m}$ injury widths, when compared to the $160 \mu \mathrm{m}$ injury width and undamaged control (Fig. 2c), suggesting the larger microinjury widths exerted the most potent effects on pre-osteoclasts.

The effect of doubly conditioned medium from microinjury induced osteoclastogenesis on pre-osteoblast migration and differentiation

Doubly conditioned medium (medium exposed to osteocytes and pre-osteoclasts) was then either directly used for pre-osteoblast migration studies or combined in a 1:1 ratio with osteoblast growth or osteogenic medium, to examine the effect on pre-osteoblast mineralisation. Pre-osteoblast migration was proportional to osteocyte network injury width, with doubly conditioned medium from a $400 \mu \mathrm{m}$ injury size showing significantly increased levels of migration, compared to the undamaged control 
a

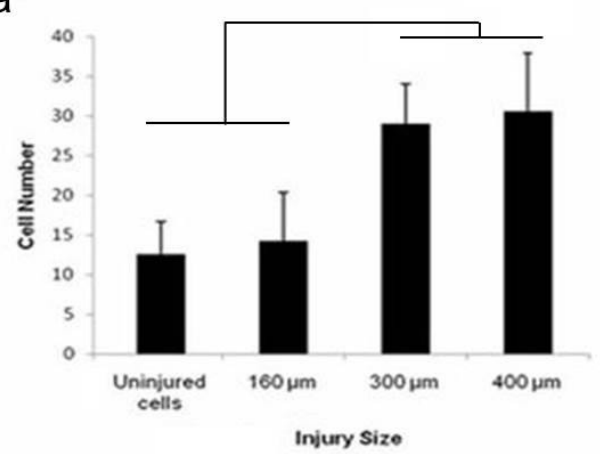

b

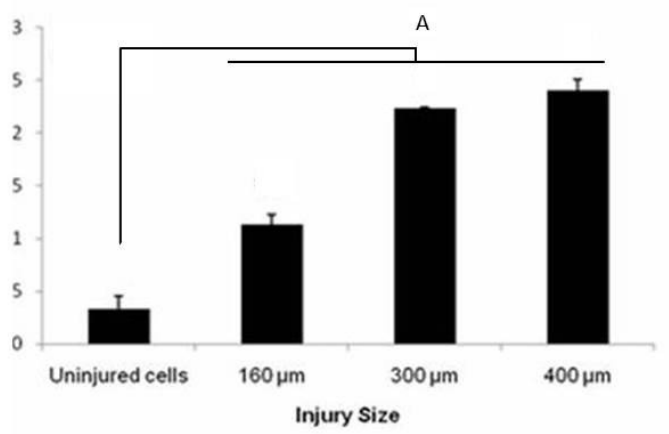

C

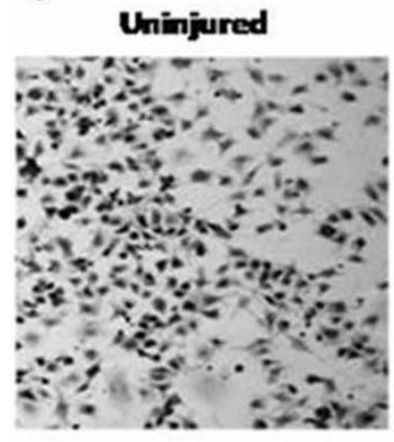

$160 \mu \mathrm{m}$

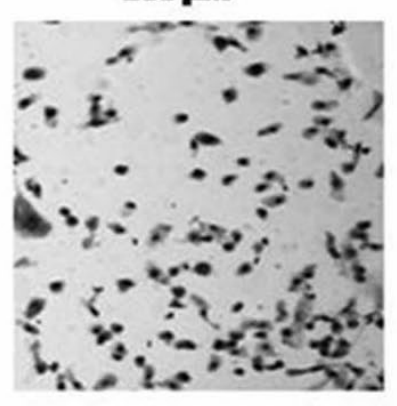

$300 \mathrm{~mm}$

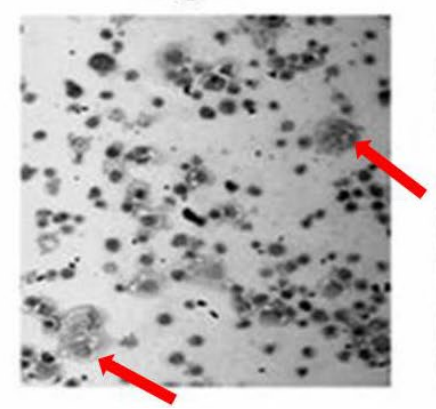

$400 \mu \mathrm{m}$

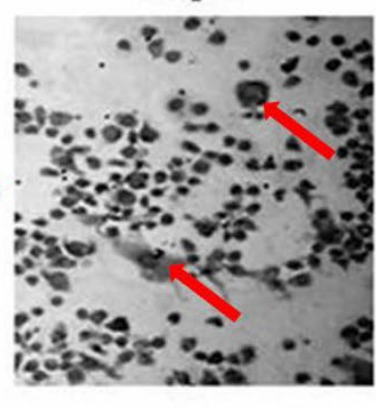

Fig. 2. The effect of injury size within gel embedded osteocyte networks on pre-osteoclast migration and differentiation. Osteocyte cultures were subjected to microinjury having widths of 160,300 or $400 \mu \mathrm{m}$. After $72 \mathrm{~h}$, conditioned medium was collected and used to assess (a) the chemotaxic properties on pre-osteoclasts, (A, $p \leq 0.05$ versus $160 \mu \mathrm{m}$ injuries and undamaged cells), (b) the induction of osteoclastogenesis after $21 \mathrm{~d}$ of culture, as quantitatively measured by TRAP activity, (A, $p \leq 0.05$ versus uninjured control), or (c) qualitatively measured by TRAP staining, which suggested the presence of large multinucleated cells was greatest in 300 and $400 \mu \mathrm{m}$ samples. Error bars represent the standard deviation, $n=3$.

(Fig. 3a). Significantly higher levels of calcification were noted in pre-osteoblasts exposed to doubly conditioned medium from $400 \mu \mathrm{m}$ injury sizes, in comparison to other injury sizes when cultured in the absence of osteogenic medium. When osteogenic medium was utilised, all injury sizes expressed similar levels of calcification (Fig. 3b). These data suggest that microinjury sizes of $400 \mu \mathrm{m}$ alone were sufficient to stimulate pre-osteoblast mineralisation.

Using an in vitro osteocyte-BMU assay system to study the effect of bisphosphonate treatment

Based on the studies described above, a $400 \mu \mathrm{m}$ injury width was the only in vitro microinjury size tested capable of inducing cellular crosstalk between the different cell types that replicated the expected in vivo response. Thus, further experiments aiming to study the role of bisphosphonates were conducted using this injury size.

The presence of bisphosphonates during osteocyte network microinjury modulates osteoclast migration and differentiation

The treatment of gel-embedded osteocyte networks with alendronate did not reduce migration of pre-osteoclasts in response to a microinjury size of $400 \mu \mathrm{m}$, whilst zoledronate surprisingly showed a significant increase in the level of pre-osteoclast migration (Fig. 4a). Interestingly, the treatment of gel-embedded osteocyte networks with alendronate or zoledronate significantly decreased osteoclastogenesis as measured by TRAP activity in undamaged controls. Microinjuries of $400 \mu \mathrm{m}$ were still capable of inducing osteoclast differentiation in bisphosphonate treated groups; however, the levels of TRAP activity (Fig. 4b) and TRAP positive cells were significantly less than that observed for the non-treated groups post microinjury (Fig. 4c).

Doubly conditioned medium from microinjury induced osteoclastogenesis under bisphosphonate treatment conditions modulates pre-osteoblast migration and differentiation

Gel-embedded osteocyte networks were subjected to a microinjury width of $400 \mu \mathrm{m}$ in the presence or absence of bisphosphonate treatment. After $72 \mathrm{~h}$, conditioned medium was collected and used in osteoclastogenesis assays (as described previously). This doubly conditioned medium was then either used for pre-osteoblast migration studies or utilised to examine the effect on pre-osteoblast mineralisation. Pre-osteoblast migration significantly increased in all treatment groups in response to microinjury, compared to undamaged controls. Significantly higher levels of cell migration were noted in the zoledronate microinjured group, in comparison to the untreated and alendronate treated microinjured groups (Fig. 5a). Bisphosphonate treatment significantly increased calcium deposition by pre-osteoblasts over a $21 \mathrm{~d}$ period, in both the undamaged and microinjured groups, with the 
a

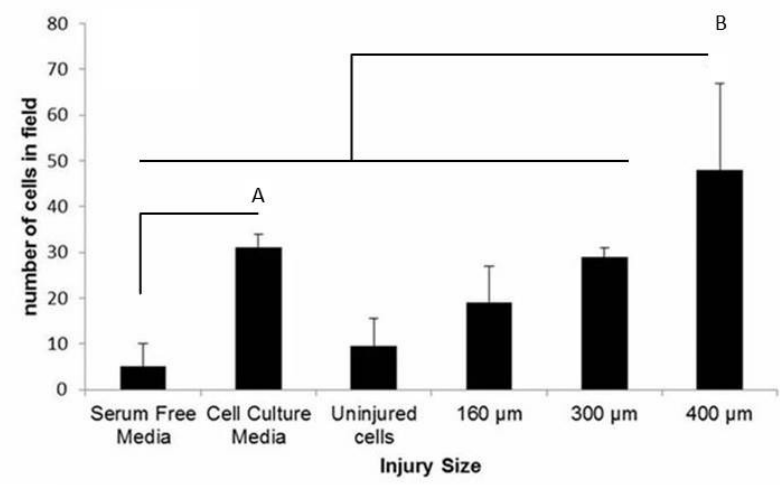

b

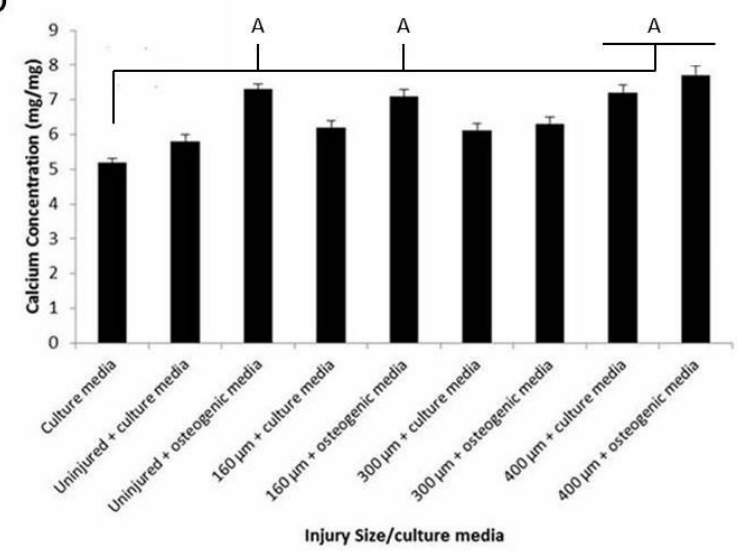

Fig. 3. The effect of microinjury induced osteoclastogenesis on pre-osteoblast migration and differentiation. Gel embedded osteocyte networks were subjected to microinjury having widths of 160,300 or $400 \mu \mathrm{m}$. After $72 \mathrm{~h}$, conditioned medium was collected and used in osteoclastogenesis assays. This doubly conditioned medium was then either directly used to evaluate preosteoblast migration (a) (A, $p \leq 0.05$ versus serum free control; $\mathrm{B}, p \leq 0.05$ versus all other groups) or combined in a 1:1 ratio with osteoblast growth or osteogenic medium and used to assess its osteogenic differentiation potential (b) (A, $p \leq 0.05$ versus cell culture medium). Error bars represent the standard deviation, $n=3$.

microinjured groups having a further significant increase in calcium deposition levels, suggesting an additive effect of microinjury with bisphosphonate treatment (Fig. 5b).

The effect of bisphosphonate treatment on the RANKLRANK-OPG pathway

Gel-embedded osteocyte networks were subjected to microinjury having an injury width of $400 \mu \mathrm{m}$ in the presence or absence of bisphosphonate treatment. Secreted RANKL and OPG protein expression levels were measured by ELISA. Bisphosphonate treatment significantly reduced both cytokines in uninjured and microinjured samples (Fig. 6a and b).

\section{Discussion}

It is well known that bone is capable of detecting and repairing microcracks and other forms of microscopic a

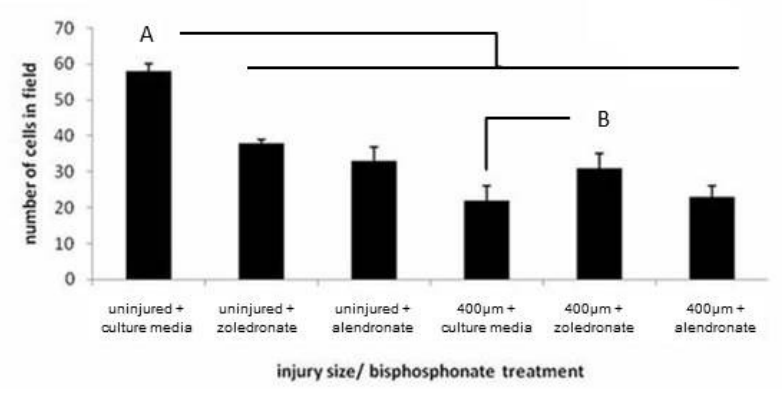

b

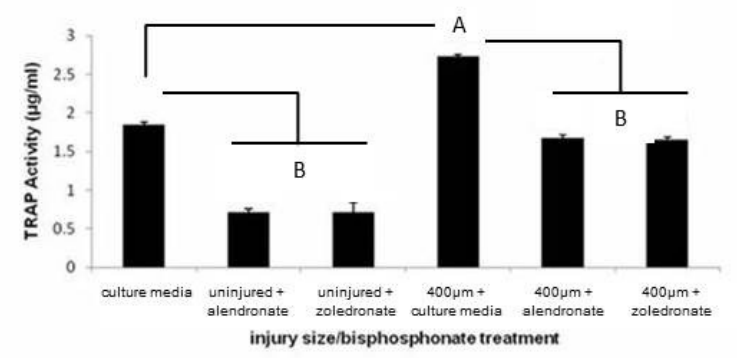

C

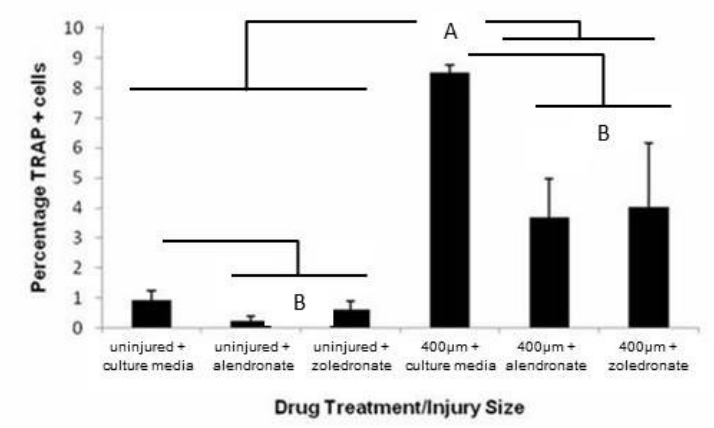

Fig. 4. The effect of microinjury within osteocyte cultures in the presence of bisphosphonates on pre-osteoclast migration and differentiation. Gel embedded osteocyte networks were subject to microinjury having a width of $400 \mu \mathrm{m}$ in the presence of alendronate or zoledronate. After $72 \mathrm{~h}$, conditioned medium was collected and used to assess either the chemotaxic properties on pre-osteoclasts (a) (A, $p \leq 0.01$ versus all other groups; $\mathrm{B}, p \leq 0.05$ versus $400 \mu \mathrm{m}$ + culture medium), or the induction of osteoclastogenesis after $21 \mathrm{~d}$ of culture, as quantitatively measured by TRAP activity (b) (A, $p \leq 0.05$ versus cell culture medium; $\mathrm{B}$, $p \leq 0.05$ decrease versus cell culture medium for same injury size) or quantification of TRAP staining (c) (A, $p \leq 0.01$ versus all other groups; $\mathrm{B}, p \leq 0.05$ decrease versus cell culture medium for same injury size). Error bars represent the standard deviation, $n=3$.

damage and that bone remodelling by BMUs is stimulated by microdamage (Burr et al., 1985; Hazenberg et al., 2009; Martin, 2002; Verborgt et al., 2000). The detection of microdamage and subsequent activation of the BMU to initiate bone remodelling is believed to be facilitated by osteocytes, as a function of their mechanosensory and mechanostimulatory attributes, either through a mechanotransduction mechanism (Turner et al., 1994) or, as hypothesised by our group, by a so called "scissors 
Fig. 5. The effect of bisphosphonate-modulated microinjury-induced osteoclastogenesis on preosteoblast migration and differentiation. Pre-osteocyte cultures were subject to microinjury having a width of $400 \mu \mathrm{m}$ in the presence of alendronate or zoledronate. After $72 \mathrm{~h}$, conditioned medium was collected and used in osteoclastogenesis assays. This doublyconditioned medium was then either directly used to evaluate pre-osteoblast migration (a) (A, $p \leq 0.05$ decrease versus all $400 \mu \mathrm{m}$ treated groups; $\mathrm{B}, p \leq 0.01$ versus $400 \mu \mathrm{m}+$ culture medium) or combined in a 1:1 ratio with osteoblast growth medium and used to assess its osteogenic differentiation potential (b) (A, $p \leq 0.05$ versus all other treatment groups except for uninjured + culture medium; $\mathrm{B}, p \leq 0.05$ versus cell culture medium group for the same injury size). Error bars represent the standard deviation, $n=3$.

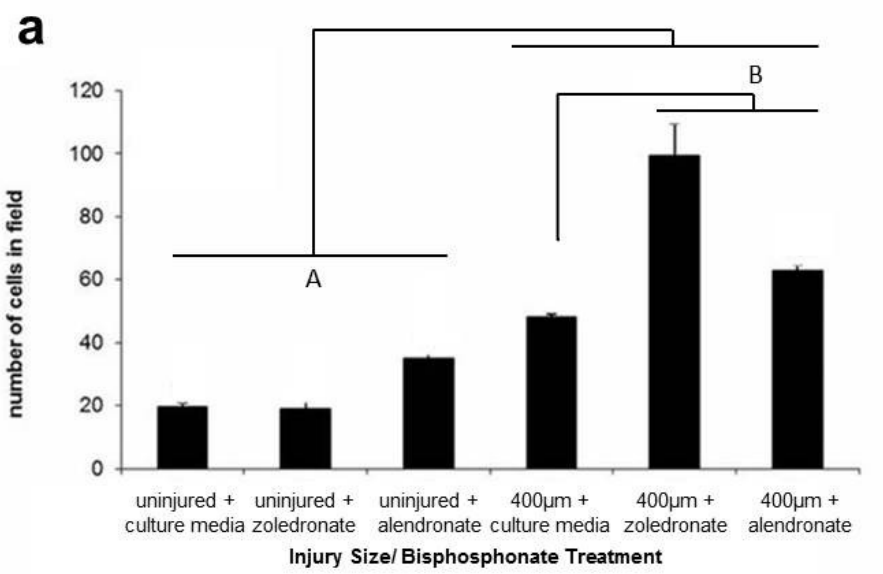

b

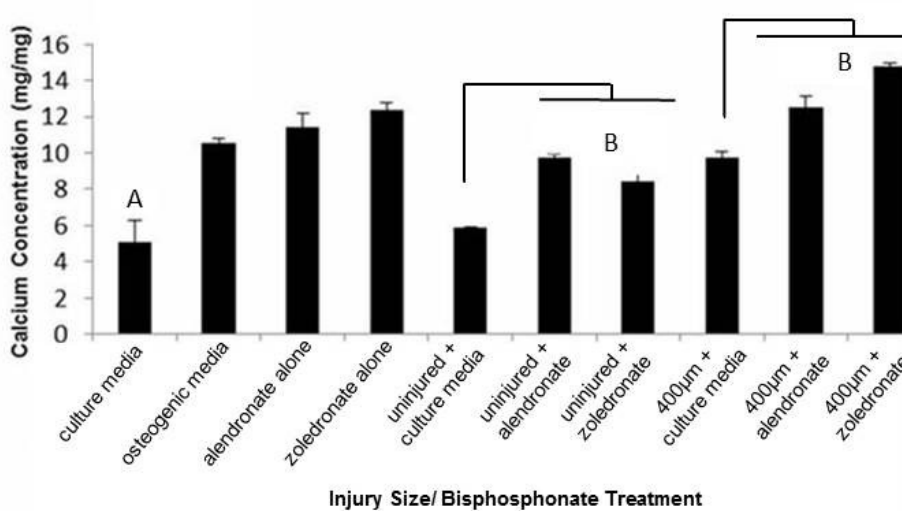

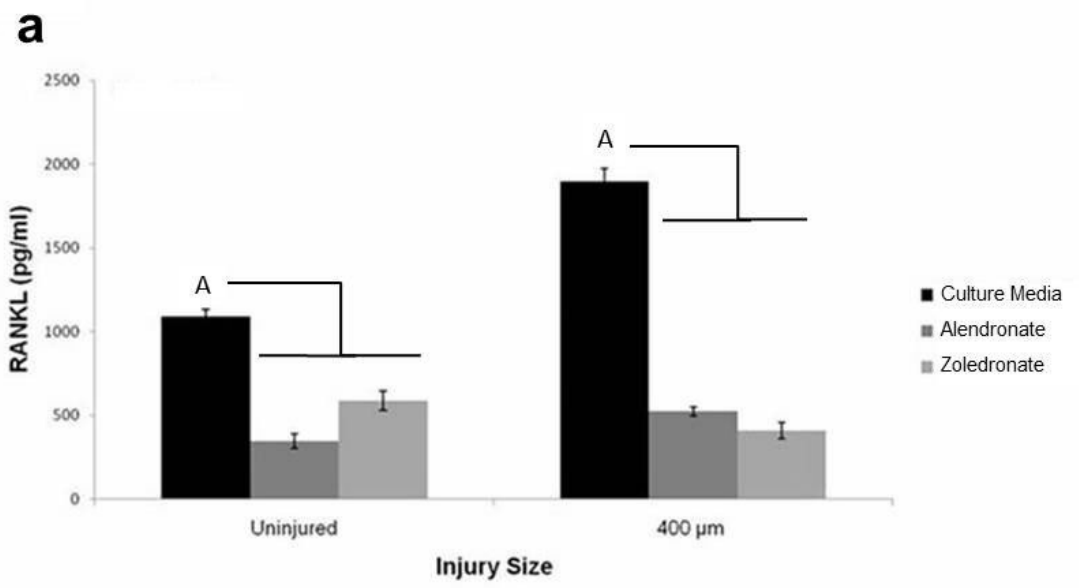

b

Fig. 6. The effect of bisphosphonate concentration on RANKL and OPG protein expression levels after osteocyte injury. Osteocyte cultures were supplemented with $10^{-5} \mathrm{M}$ alendronate or zoledronate concentrations and subject to microinjury having a width of $400 \mu \mathrm{m}$. RANKL (a) and OPG (b) protein expression levels were quantified by ELISA (A, $p \leq 0.05$ versus bisphosphonate-treated groups for the same injury size). Error bars represent the standard deviation, $n=3$.

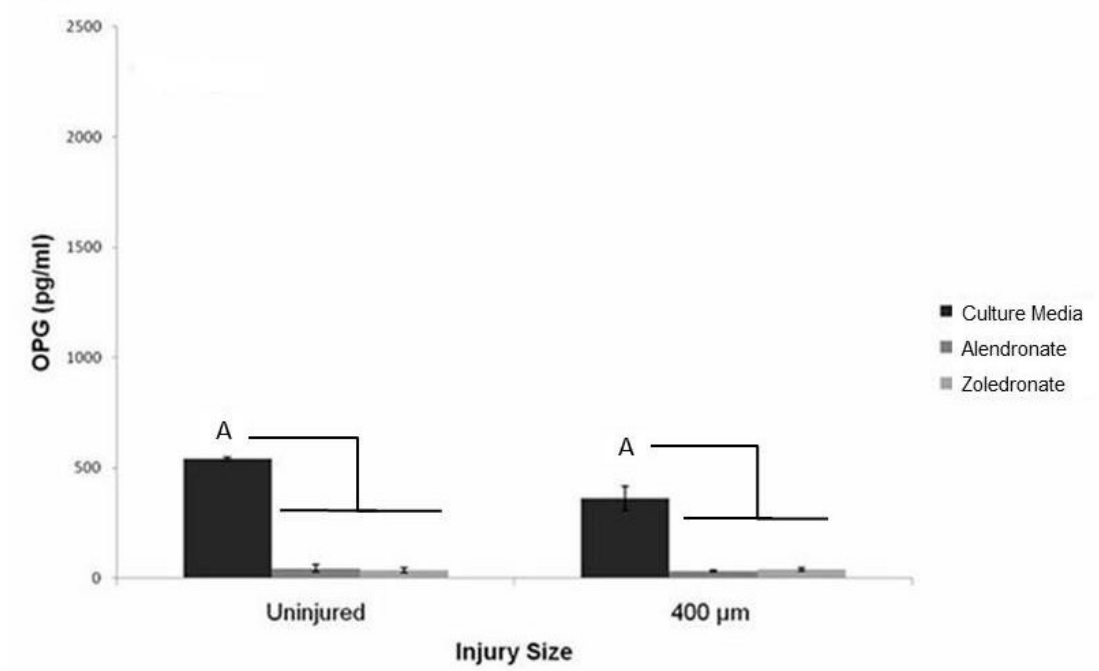


model" that involves the shearing of cellular processes (Hazenberg et al., 2009; Taylor et al., 2003). In order to study this behaviour in vitro, a model allowing controlled levels of injury and damage to osteocyte networks was developed in-house, in the absence of the bone tissue matrix (Mulcahy et al., 2011). This study showed that this in vitro microinjury can modulate the RANKL-RANKOPG signalling pathway (Mulcahy et al., 2011) in a manner indicative of the in vivo response, which is associated with the initiation of bone remodelling through the synchronous action of the BMU. Bone remodelling consists of several stages, which include activation, resorption and formation. Activation refers to the stage by which an influx of osteoclast precursor cells infiltrates the area of osteocyte rupture and fuse to form giant, active osteoclasts. These differentiated osteoclasts then polarise to bone and release lytic enzymes, which break down bone, under the control of RANKL-RANK interaction (Katagiri and Takahashi, 2002). Finally, formation of new bone, by the infiltration of pre-osteoblasts, leads to the secretion of osteoid, which becomes mineralised by calcium and phosphates in peripheral fluids. This occurs following release of OPG, which stops RANKL-RANK interaction, and stimulates the influx of pre-osteoblasts.

This study describes the successful development of an in vitro model capable of mimicking this interactive crosstalk between sensory osteocytic networks and the osteoclastogenic and osteogenic activities of the BMU. We showed that in response to microinjury sizes of $400 \mu \mathrm{m}$, pre-osteoclast migration was increased, and osteoclast differentiation, as measured by TRAP activity, was significantly upregulated. Furthermore, doublyconditioned medium was capable of enhancing preosteoblast migration and pre-osteoblast differentiation, as measured by the deposition of calcium.

In vitro models, as developed in this study, offer researchers a potential means by which complex systems such as bone remodelling can be decoupled and the effects of pharmaceutical agents on individual components of the system can be analysed. For example, since the early 1990s bisphosphonates have yielded significant potential and have shown great success in the treatment of osteoporosis, as well as other bone diseases, including metastatic bone disease, osteopenia and Paget's disease (Morris and Einhorn, 2005). In spite of the existing knowledge of the in vivo responses of bone to bisphosphonates, it is well recognised that the cellular responses to these drugs require further investigation. In this study, an in vitro model system was applied to investigate the effect of two aminobisphosphonates, alendronate and zoledronate, on osteocyte stimulation in response to microinjury and the subsequent impact upon the cells constituting the BMU.

The changes observed in the RANKL-RANK-OPG signalling pathway, as a function of bisphosphonate treatment, directly resulted in reduced osteoclast activity as measured by TRAP activity, as observed by other groups previously in vitro (Boonekamp et al., 1986; Kimachi et al., 2011; Li et al., 2011), in line with the expected method of action for these drugs in vivo. However, previous in vivo studies have suggested that alendronate is a less potent inhibitor of osteoclast activity than zoledronate (Watts and
Diab, 2010), though the results herein suggest there is no difference between the two bisphosphonates when tested in vitro. It is important to point out that the differences observed in inhibition of osteoclast activity in vivo relies on a number of factors that primarily includes the method of bisphosphonate administration (i.e. intravenous versus oral) and also the different bioavailability. For example, oral bisphosphonates have very limited bioavailability due to their poor lipophilicity, with less than $1 \%$ of orally taken bisphosphonates being absorbed in the gut (Ruza et al., 2013). As a result, the potency is also different but in a comparable order of magnitude. This was demonstrated in a study by Orwoll et al. (2010) that showed similar increased bone density and decreased bone turnover markers when comparing the safety and efficacy of a once-yearly intravenous infusion of $5 \mathrm{mg}$ zoledronic acid versus a once-weekly $70 \mathrm{mg}$ oral alendronate in the treatment of male osteoporosis. The effect of bisphosphonate treatment on pre-osteoblast migration and differentiation was subsequently evaluated. Both zoledronate and alendronate significantly increased both pre-osteoblast migration and differentiation in response to microinjury. These findings are in line with previous reports suggesting that aminobisphosphonates, including alendronate and zoledronate, have stimulatory effects on osteoblast differentiation and proliferation (Pan et al., 2004; Reinholz et al., 2000). Although the exact mechanism by which bisphosphonates affect osteoblasts remains elusive, zoledronic acid has been shown to inhibit the mevalonate pathway thereby inducing human osteoblast differentiation (Reinholz et al., 2002). Inhibition of this pathway also prevents osteoclasts from attaching to the bone surface and therefore resorbing bone (Fisher et al., 2000).

This study also showed that the release of two of the principal cytokines involved in bone remodelling, RANKL, which functions by stimulating osteoclastogenesis, and its antagonist, OPG, which sequesters RANKL activity, stimulating osteoblastogenesis (Lacey et al., 1998; Simonet et al., 1997) were significantly reduced in response to bisphosphonate treatments. These results are reflective of the work of others (Giner et al., 2011; Kimachi et al., 2011; Koch et al., 2012), who observed that aminobisphosphonates, including alendronate and zoledronate, posed a modulatory effect on RANKL activity in osteoblastic cells. Considering that in this model the effect of bisphosphonates on an osteocytic network is being assessed, the results may be significant in further demonstrating the biochemical effect of osteoporotic drugs, most notably so in a model of microinjury in bone. However, the reduction in OPG, in both alendronate and zoledronate-treated samples, is something that has not been extensively observed to date and provides further mechanistic information regarding the action of such drugs.

In conclusion, the in vivo effects of bisphosphonates are, at present, well documented, and treatment of osteoporosis using bisphosphonates has shown great promise with regard to decreasing osteoclast activity and increasing bone mineral density. However, the cellular response to bisphosphonates, in an environment mimicking microinjury of the osteocyte network, has yet to be clarified. This study, using a novel in vitro model of bone remodelling, which 
allowed the spatial decoupling of the different cell types involved, has shown for the first time that microinjury, under the treatment of aminobisphosphonates, has a modulatory effect on RANKL and OPG activity, and thus on osteoclastogenesis. Furthermore, the presence of bisphosphonates was shown to increase the osteogenic potential of microinjury. Thus, we have shown that bisphosphonates act at all three stages of bone remodelling, from microinjury to osteoclastogenesis and ultimately osteogenesis.

\section{Acknowledgments}

This work was funded by Science Foundation Ireland under the Research Frontiers Programme grant number RFPENM-991. The MLO-Y4 cells were generously provided by Dr. Linda Bonewald (School of Dentistry, University of Missouri, Kansas City, MO, USA). Authors' roles: Planning: LM, GD, TCL and DT. Data collection: LM. Data analysis: LM, CC, RM and GD. Manuscript preparation and editing: LM, RM, CC, GD, DT and TCL. GD takes responsibility for the integrity of the data analysis. We wish to confirm that there are no known conflicts of interest associated with this publication and there has been no significant financial support for this work that could have influenced its outcome.

\section{References}

Al-Dujaili SA, Lau E, Al-Dujaili H, Tsang K, Guenther A, You L (2011) Apoptotic osteocytes regulate osteoclast precursor recruitment and differentiation in vitro. $\mathrm{J}$ Cell Biochem 112: 2412-2423.

Bergmann P, Body JJ, Boonen S, Boutsen Y, Devogelaer JP, Goemaere S, Kaufman J, Reginster JY, Rozenberg S (2010) Loading and skeletal development and maintenance. J Osteoporos 2011: 786752.

Boivin GY, Chavassieux PM, Santora AC, Yates J, Meunier PJ (2000) Alendronate increases bone strength by increasing the mean degree of mineralization of bone tissue in osteoporotic women. Bone 27: 687-694.

Boonekamp PM, van der Wee-Pals LJ, van Wijk-van Lennep MM, Thesing CW, Bijvoet OL (1986) Two modes of action of bisphosphonates on osteoclastic resorption of mineralized matrix. Bone Miner 1: 27-39.

Brennan O, Kennedy OD, Lee TC, Rackard SM, O'Brien FJ, McNamara LM (2011) The effects of estrogen deficiency and bisphosphonate treatment on tissue mineralisation and stiffness in an ovine model of osteoporosis. J Biomech 44: 386-390.

Burge R, Dawson-Hughes B, Solomon DH, Wong JB, King A, Tosteson A (2007) Incidence and economic burden of osteoporosis-related fractures in the United States, 20052025. J Bone Miner Res 22: 465-475.

Burr DB, Martin RB, Schaffler MB, Radin EL (1985) Bone remodeling in response to in vivo fatigue microdamage. J Biomech 18: 189-200.

Burr DB, Milgrom C, Fyhrie D, Forwood M, Nyska M, Finestone A, Hoshaw S, Saiag E, Simkin A (1996) In vivo measurement of human tibial strains during vigorous activity. Bone 18: 405-410.

Burr DB, Turner CH, Naick P, Forwood MR, Ambrosius W, Hasan MS, Pidaparti R (1998) Does microdamage accumulation affect the mechanical properties of bone? $\mathrm{J}$ Biomech 31: 337-345.

Chrischilles E, Shireman T, Wallace R (1994) Costs and health effects of osteoporotic fractures. Bone 15: 377-386.

Donahue SW, Galley SA, Vaughan MR, PattersonBuckendahl P, Demers LM, Vance JL, McGee ME (2006) Parathyroid hormone may maintain bone formation in hibernating black bears (Ursus americanus) to prevent disuse osteoporosis. J Exp Biol 209: 1630-1638.

Eriksen EF, Langdahl B, Vesterby A, Rungby J, Kassem M (1999) Hormone replacement therapy prevents osteoclastic hyperactivity: A histomorphometric study in early postmenopausal women. J Bone Miner Res 14: 12171221.

Fisher JE, Rodan GA, Reszka AA (2000) In vivo effects of bisphosphonates on the osteoclast mevalonate pathway. Endocrinology 141: 4793-4796.

Giner M, Rios MJ, Montoya MJ, Vazquez MA, Miranda C, Perez-Cano R (2011) Alendronate and raloxifene affect the osteoprotegerin/RANKL system in human osteoblast primary cultures from patients with osteoporosis and osteoarthritis. Eur J Pharmacol 650: 682-687.

Hazenberg JG, Hentunen TA, Heino TJ, Kurata K, Lee TC, Taylor D (2009) Microdamage detection and repair in bone: fracture mechanics, histology, cell biology. Technol Health Care 17: 67-75.

Hoerger TJ, Downs KE, Lakshmanan MC, Lindrooth RC, Plouffe L, Jr., Wendling B, West SL, Ohsfeldt RL (1999) Healthcare use among U.S. women aged 45 and older: total costs and costs for selected postmenopausal health risks. J Womens Health Gend-Based Med 8: 1077 1089.

Hoey DA, Kelly DJ, Jacobs CR (2011) A role for the primary cilium in paracrine signaling between mechanically stimulated osteocytes and mesenchymal stem cells. Biochem Biophys Res Commun 412:182-187.

Jilka RL, Takahashi K, Munshi M, Williams DC, Roberson PK, Manolagas SC (1998) Loss of estrogen upregulates osteoblastogenesis in the murine bone marrow. Evidence for autonomy from factors released during bone resorption. J Clin Invest 101: 1942-1950.

Johnell O, Kanis JA (2006) An estimate of the worldwide prevalence and disability associated with osteoporotic fractures. Osteoporos Int 17: 1726-1733.

Katagiri T, Takahashi N (2002) Regulatory mechanisms of osteoblast and osteoclast differentiation. Oral Dis 8: 147-159.

Kato Y, Windle JJ, Koop BA, Mundy GR, Bonewald LF (1997) Establishment of an osteocyte-like cell line, MLO-Y4. J Bone Miner Res 12: 2014-2023.

Kimachi K, Kajiya H, Nakayama S, Ikebe T, Okabe K (2011) Zoledronic acid inhibits RANK expression and migration of osteoclast precursors during osteoclastogenesis. Naunyn Schmiedebergs Arch Pharmacol 383: 297-308.

Koch FP, Merkel C, Ziebart T, Smeets R, Walter C, Al-Nawas B (2012) Influence of bisphosphonates on the 
osteoblast RANKL and OPG gene expression in vitro. Clin Oral Invest 16: 79-86.

Kufahl RH, Saha S (1990) A theoretical model for stress-generated fluid flow in the canaliculi-lacunae network in bone tissue. J Biomech 23: 171-180.

Lacey DL, Timms E, Tan HL, Kelley MJ, Dunstan CR, Burgess T, Elliott R, Colombero A, Elliott G, Scully S, Hsu H, Sullivan J, Hawkins N, Davy E, Capparelli C, Eli A, Qian YX, Kaufman S, Sarosi I, Shalhoub V, Senaldi G, Guo J, Delaney J, Boyle WJ (1998) Osteoprotegerin ligand is a cytokine that regulates osteoclast differentiation and activation. Cell 93: 165-176.

Lanyon LE (1987) Functional strain in bone tissue as an objective, and controlling stimulus for adaptive bone remodelling. J Biomech 20: 1083-1093.

Lee TC, Staines A, Taylor D (2002) Bone adaptation to load: microdamage as a stimulus for bone remodelling. J Anat 201: 437-446.

Li B, Ling Chau JF, Wang X, Leong WF (2011) Bisphosphonates, specific inhibitors of osteoclast function and a class of drugs for osteoporosis therapy. J Cell Biochem 112: 1229-1242.

Martin RB (2002) Is all cortical bone remodeling initiated by microdamage? Bone 30: $8-13$.

Martin RB, Burr DB (1982) A hypothetical mechanism for the stimulation of osteonal remodelling by fatigue damage. J Biomech 15: 137-139.

Mashiba T, Hirano T, Turner CH, Forwood MR, Johnston CC, Burr DB (2000) Suppressed bone turnover by bisphosphonates increases microdamage accumulation and reduces some biomechanical properties in dog rib. J Bone Miner Res 15: 613-620.

Morris CD, Einhorn TA (2005) Bisphosphonates in orthopaedic surgery. J Bone Joint Surg Am 87: 1609-1618.

Mulcahy LE, Taylor D, Lee TC, Duffy GP (2011) RANKL and OPG activity is regulated by injury size in networks of osteocyte-like cells. Bone 48: 182-188.

Naidu A, Dechow PC, Spears R, Wright JM, Kessler HP, Opperman LA (2008) The effects of bisphosphonates on osteoblasts in vitro. Oral Surg Oral Med Oral Pathol Oral Radiol Endod 106: 5-13.

Nordin BE, Need AG, Chatterton BE, Horowitz M, Morris HA (1990) The relative contributions of age and years since menopause to postmenopausal bone loss. J Clin Endocrinol Metab 70: 83-88.

Orwoll ES, Miller PD, Adachi JD, Brown J, Adler RA, Kendler D, Bucci-Rechtweg C, Readie A, Mesenbrink P, Weinstein RS (2010) Efficacy and safety of a once-yearly i.v. Infusion of zoledronic acid $5 \mathrm{mg}$ versus a onceweekly 70-mg oral alendronate in the treatment of male osteoporosis: A randomized, multicenter, double-blind, active-controlled study J Bone Miner Res 25: 2239-2250.

Pan B, To LB, Farrugia AN, Findlay DM, Green J, Gronthos S, Evdokiou A, Lynch K, Atkins GJ, Zannettino AC (2004) The nitrogen-containing bisphosphonate, zoledronic acid, increases mineralisation of human bonederived cells in vitro. Bone 34: 112-123.
Parfitt AM (2002) High bone turnover is intrinsically harmful: two paths to a similar conclusion. The Parfitt view. J Bone Miner Res 17: 1558-1559.

Phillips S, Fox N, Jacobs J, Wright WE (1988) The direct medical costs of osteoporosis for American women aged 45 and older, 1986. Bone 9: 271-279.

Pivonka P, Buenzli PR, Dunstan CR (2012). A systems approach to understanding bone cell interactions in health and disease. In: Gowder S (ed.) Cell Interaction, InTech, Rijeka, Croatia, pp 169-204.

Ray NF, Chan JK, Thamer M, Melton LJ 3rd (1997) Medical expenditures for the treatment of osteoporotic fractures in the United States in 1995: report from the National Osteoporosis Foundation. J Bone Miner Res 12: 24-35.

Reginster JY, Pelousse F, Bruyere O (2013) Safety concerns with the long-term management of osteoporosis. Expert Opin Drug Saf 12: 507-522.

Reinholz GG, Getz B, Pederson L, Sanders ES, Subramaniam M, Ingle JN, Spelsberg TC (2000) Bisphosphonates directly regulate cell proliferation, differentiation, and gene expression in human osteoblasts. Cancer Res 60: 6001-6007.

Reinholz GG, Getz B, Sanders ES, Karpeisky MY, Padyukova N, Mikhailov SN, Ingle JN, Spelsberg TC (2002) Distinct mechanisms of bisphosphonate action between osteoblasts and breast cancer cells: identity of a potent new bisphosphonate analogue. Breast Cancer Res Treat 71: 257-268.

Roche JJ, Wenn RT, Sahota O, Moran CG (2005) Effect of comorbidities and postoperative complications on mortality after hip fracture in elderly people: prospective observational cohort study. BMJ 331: 1374.

Rogers MJ (2003) New insights into the molecular mechanisms of action of bisphosphonates. Curr Pharm Des 9: 2643-2658.

Russell RG, Watts NB, Ebetino FH, Rogers MJ (2008) Mechanisms of action of bisphosphonates: similarities and differences and their potential influence on clinical efficacy. Osteoporos Int 19: 733-759.

Ruza I, Mirfakhraee S, Orwoll E, Gruntmanis U (2013) Clinical experience with intravenous zoledronic acid in the treatment of male osteoporosis: evidence and opinions. Ther Adv Musculoskelet Dis 5: 182-198.

Simonet WS, Lacey DL, Dunstan CR, Kelley M, Chang MS, Luthy R, Nguyen HQ, Wooden S, Bennett L, Boone T, Shimamoto G, DeRose M, Elliott R, Colombero A, Tan HL, Trail G, Sullivan J, Davy E, Bucay N, Renshaw-Gegg L, Hughes TM, Hill D, Pattison W, Campbell P, Sander S, Van G, Tarpley J, Derby P, Lee R, Boyle WJ (1997) Osteoprotegerin: a novel secreted protein involved in the regulation of bone density. Cell 89: 309-319.

Spadaro JA, Damron TA, Horton JA, Margulies BS, Murray GM, Clemente DA, Strauss JA (2006) Density and structural changes in the bone of growing rats after weekly alendronate administration with and without a methotrexate challenge. J Orthop Res 24: 936-944.

Taylor D, Hazenberg JG, Lee TC (2003) The cellular transducer in damage-stimulated bone remodelling: a 
theoretical investigation using fracture mechanics. J Theor Biol 225: 65-75.

Turner CH, Forwood MR, Otter MW (1994) Mechanotransduction in bone: do bone cells act as sensors of fluid flow? FASEB J 8: 875-878.

Verborgt O, Gibson GJ, Schaffler MB (2000) Loss of osteocyte integrity in association with microdamage and bone remodeling after fatigue in vivo. J Bone Miner Res 15: $60-67$.

Watts NB, Diab DL (2010) Long-term use of bisphosphonates in osteoporosis. J Clin Endocrinol Metab 95: 1555-1565.

Weinbaum S, Cowin SC, Zeng Y (1994) A model for the excitation of osteocytes by mechanical loading-induced bone fluid shear stresses. J Biomech 27: 339-360.

Zerwekh JE, Ruml LA, Gottschalk F, Pak CY (1998) The effects of twelve weeks of bed rest on bone histology, biochemical markers of bone turnover, and calcium homeostasis in eleven normal subjects. J Bone Miner Res 13: $1594-1601$.

\section{Discussion with Reviewer}

Reviewer I: For this model, why did the authors not co-culture the osteoblasts and osteoclasts together from the beginning in one dish or plate and then transfer the microdamage conditioned media?

Authors: In order to test this model at the most basic level, we chose to assess the individual cell types to gain a basic understanding of how the individual cell types function during bone remodelling. It was agreed that were a coculture to be evaluated it would introduce a further level of complexity that was beyond the scope of this particular study but may be of interest in future studies.

Editor's Note: Scientific Editor in charge of the paper: Martin Stoddart. 